\title{
COMPLEMENTARY RESULTS TO HEUVERS'S CHARACTERIZATION OF LOGARITHMIC FUNCTIONS
}

\author{
MARTin Himmel
}

\begin{abstract}
Based on a characterization of logarithmic functions due to Heuvers we develop analogous results for multiplicative, exponential and additive functions, respectively.
\end{abstract}

\section{Introduction}

Heuvers [1] (see also Kannappan [3, p. 31]), using a cocycle equation, gave the following characterization of logarithmic functions:

a function $f:(0, \infty) \rightarrow \mathbb{R}$ satisfies the functional equation

$$
f(x+y)-f(x)-f(y)=f\left(\frac{1}{x}+\frac{1}{y}\right), \quad x, y>0,
$$

if and only if

$$
f(x y)=f(x)+f(y), \quad x, y>0 .
$$

On this basis, we present analogous characterizations for the remaining three Cauchy equations, i.e. for multiplicative, exponential and additive functions. In the case of exponential and additive functions the classical log function appears.

Received: 08.10.2016. Accepted: 26.11.2016. Published online: 17.02.2017.

(2010) Mathematics Subject Classification: 26E60, 39B22, 97I70.

Key words and phrases: means, functional equation. 
Let us observe that Heuvers' result can be formulated also in terms of the Cauchy difference $C_{f}:(0, \infty)^{2} \rightarrow \mathbb{R}$,

$$
C_{f}(x, y):=f(x+y)-f(x)-f(y), \quad x, y>0,
$$

of a generator $f:(0, \infty) \rightarrow \mathbb{R}$, and the harmonic mean $H:(0, \infty)^{2} \rightarrow(0, \infty)$,

$$
H(x, y)=\frac{2 x y}{x+y}, \quad x, y>0 .
$$

Remark $1([2])$. The generator $f:(0, \infty) \rightarrow \mathbb{R}$ of the Cauchy difference satisfies the functional equation

$$
C_{f}(x, y)=f\left(\frac{2}{H(x, y)}\right), \quad x, y>0
$$

if and only if $f$ is a logarithmic function, i.e.

$$
f(x y)=f(x)+f(y), \quad x, y>0 .
$$

\section{Characterization of multiplicative functions}

The following result characterizes multiplicative functions.

THEOREM 1. The function $g:(0, \infty) \rightarrow(0, \infty)$ satisfies the functional equation

$$
\frac{g(x+y)}{g(x) g(y)}=g\left(\frac{1}{x}+\frac{1}{y}\right), \quad x, y>0,
$$

if and only if $g$ is a multiplicative function.

Proof. Assume that $g:(0, \infty) \rightarrow(0, \infty)$ satisfies (2.1). Taking the logarithm of both sides, we obtain

$$
\log \left(\frac{g(x+y)}{g(x) g(y)}\right)=\log \left(g\left(\frac{1}{x}+\frac{1}{y}\right)\right), \quad x, y>0,
$$


and thus, using properties of the function $\log :(0, \infty) \rightarrow \mathbb{R}$, we have

$$
\log (g(x+y))-\log (g(x))-\log (g(y))=\log \left(g\left(\frac{1}{x}+\frac{1}{y}\right)\right), \quad x, y>0 .
$$

Due to Heuvers' result, the function $f:=\log \circ g$ is logarithmic, i.e.

$$
\log (g(x y))=\log (g(x))+\log (g(y)), \quad x, y>0
$$

whence

$$
\log (g(x y))=\log (g(x) g(y)), \quad x, y>0,
$$

and the injectivity of the function log implies that

$$
g(x y)=g(x) g(y), \quad x, y>0 .
$$

To prove the converse implication, assume that $g:(0, \infty) \rightarrow(0, \infty)$ is multiplicative.

Since $g$ is positive, the multiplicativity implies that

$$
g(1)=1
$$

and

$$
g\left(\frac{1}{x}\right)=\frac{1}{g(x)}, \quad x>0
$$

Hence, we have for all $x, y>0$,

$$
\begin{aligned}
g\left(\frac{1}{x}+\frac{1}{y}\right) & =g\left((x+y) \frac{1}{x y}\right)=g(x+y) g\left(\frac{1}{x y}\right) \\
& =\frac{g(x+y)}{g(x y)}=\frac{g(x+y)}{g(x) g(y)},
\end{aligned}
$$

which completes the proof.

Motivated by the relationship between the Euler Gamma function and the Beta function, let us introduce the following 
Definition 1 ([2]). For arbitrary $g:(0, \infty) \rightarrow(0, \infty)$ we call $B_{g}:(0, \infty)^{2} \rightarrow$ $(0, \infty)$ defined by

$$
B_{g}(x, y)=\frac{g(x) g(y)}{g(x+y)}, \quad x, y>0
$$

the beta-type function of generator $g$.

Note that for any function $g:(0, \infty) \rightarrow(0, \infty)$ we have

$$
B_{\frac{1}{g}}=\frac{1}{B_{g}} .
$$

This fact, Theorem 1 and the definition of beta-type functions allow us to formulate the following

REMARK 2. The generator $g:(0, \infty) \rightarrow(0, \infty)$ of the beta-type function $B_{\frac{1}{g}}:(0, \infty)^{2} \rightarrow(0, \infty)$ satisfies the functional equation

$$
B_{\frac{1}{g}}(x, y)=g\left(\frac{2}{H(x, y)}\right), \quad x, y>0
$$

if and only if $g$ is a multiplicative function, i.e.

$$
g(x y)=g(x) g(y), \quad x, y>0 .
$$

This shows that the harmonic mean is indirectly related to the Beta function (cf. [2]).

\section{Characterization of exponential functions}

From Theorem 1 we get the following.

Corollary 1. A function $h: \mathbb{R} \rightarrow(0, \infty)$ is exponential, i.e.

$$
h(s+t)=h(s) h(t), \quad s, t \in \mathbb{R},
$$

if and only if

$$
\frac{h \circ \log (x+y)}{(h \circ \log (x))(h \circ \log (y))}=h \circ \log \left(\frac{1}{x}+\frac{1}{y}\right), \quad x, y>0 .
$$


Proof. Assume that $h$ is exponential. Hence, using properties of the function $\log :(0, \infty) \rightarrow \mathbb{R}$, we have, for all $x, y>0$,

$$
\begin{aligned}
\left(h \circ \log \left(\frac{1}{x}+\frac{1}{y}\right)\right) & (h \circ \log x)(h \circ \log y) \\
& =\left(h \circ \log \left(\frac{1}{x}+\frac{1}{y}\right)\right)(h \circ(\log x+\log y)) \\
& =\left(h \circ \log \left(\frac{1}{x}+\frac{1}{y}\right)\right)(h \circ \log (x y)) \\
& =h\left(\log \left(\frac{1}{x}+\frac{1}{y}\right)+\log (x y)\right) \\
& =h\left(\log \left(\left(\frac{1}{x}+\frac{1}{y}\right) x y\right)\right)=h(\log (y+x)) \\
& =h \circ \log (x+y),
\end{aligned}
$$

thus (3.1) holds.

Conversely, assume that $h$ satisfies (3.1), and put $g:=h \circ \log$. Then $h=g \circ \exp$ and, by (3.1),

$$
\frac{g \circ \exp \circ \log (x+y)}{(g \circ \exp \circ \log (x))(g \circ \exp \circ \log (y))}=g \circ \exp \circ \log \left(\frac{1}{x}+\frac{1}{y}\right), \quad x, y>0 .
$$

Hence, using properties of the functions exp and log, we have

$$
\frac{g(x+y)}{g(x) g(y)}=g\left(\frac{1}{x}+\frac{1}{y}\right), \quad x, y>0 .
$$

By Theorem 1, the function $g$ is multiplicative, i.e.

$$
g(x y)=g(x) g(y), \quad x, y>0 .
$$

For all $x, y>0$ there exist $s, t \in \mathbb{R}$ such that

$$
x=e^{s}, \quad y=e^{t} .
$$

Thus, by the multiplicativity of $g$,

$$
g\left(e^{s+t}\right)=g\left(e^{s}\right) g\left(e^{t}\right), \quad s, t \in \mathbb{R},
$$


which means that the function $h=g \circ \exp$ is exponential. This finishes the proof.

In the spirit of the preceeding two remarks, we have the following.

REmark 3. A function $h: \mathbb{R} \rightarrow(0, \infty)$ is exponential if and only if

$$
B_{\frac{1}{h \circ \log }}(x, y)=h \circ \log \left(\frac{2}{H(x, y)}\right), \quad x, y>0 .
$$

\section{Characterization of additive functions}

The characterization of additive functions with the aid of Heuvers' result is given in the following.

Corollary 2. A function $\alpha: \mathbb{R} \rightarrow \mathbb{R}$ is additive, i.e.

$$
\alpha(s+t)=\alpha(s)+\alpha(t), \quad s, t \in \mathbb{R},
$$

if and only if

(4.1) $\alpha \circ \log (x+y)-\alpha \circ \log (x)-\alpha \circ \log (y)=\alpha \circ \log \left(\frac{1}{x}+\frac{1}{y}\right), \quad x, y>0$.

Proof. Assume that $\alpha$ is additive. Using this and properties of the function $\log$, we have, for all $x, y>0$,

$$
\begin{aligned}
\alpha \circ \log (x+y) & -\alpha \circ \log (x)-\alpha \circ \log (y)-\alpha \circ \log \left(\frac{1}{x}+\frac{1}{y}\right) \\
& =\alpha\left(\log (x+y)-\log x-\log y-\log \left(\frac{1}{x}+\frac{1}{y}\right)\right) \\
& =\alpha\left(\log \left((x+y) \frac{1}{x} \frac{1}{y} \frac{1}{\left(\frac{1}{x}+\frac{1}{y}\right)}\right)\right) \\
& =\alpha(\log 1)=\alpha(0)=0,
\end{aligned}
$$

which shows 4.1. 
Vice versa, assume that $\alpha$ satisfies (4.1). Put $h:=\alpha \circ \log$. Hence, $\alpha=h \circ \exp$ and, by (4.1), using properties of the functions exp and log, we have, for all $x, y>0$,

$$
\begin{aligned}
\alpha \circ \log (x+y) & -\alpha \circ \log (x)-\alpha \circ \log (y) \\
& =h \circ \exp \circ \log (x+y)-h \circ \exp \circ \log (x)-h \circ \exp \circ \log (y) \\
& =h \circ \exp \circ \log \left(\frac{1}{x}+\frac{1}{y}\right) \\
& =\alpha \circ \log \left(\frac{1}{x}+\frac{1}{y}\right)
\end{aligned}
$$

thus

$$
h(x+y)-h(x)-h(y)=h\left(\frac{1}{x}+\frac{1}{y}\right), \quad x, y>0 .
$$

Applying the function exp to both sides and using its properties we obtain

$$
\frac{e^{h(x+y)}}{e^{h(x)} e^{h(y)}}=e^{h\left(\frac{1}{x}+\frac{1}{y}\right)}, \quad x, y>0 .
$$

Thus, by the definition of $h$, the function $g:=\exp \circ \alpha \circ \log$ satisfies

$$
\frac{g(x+y)}{g(x) g(y)}=g\left(\frac{1}{x}+\frac{1}{y}\right), \quad x, y>0,
$$

and Theorem 1 implies that $g$ is multiplicative, i.e.

$$
g(x y)=g(x) g(y), \quad x, y>0 .
$$

Applying here the function log to both sides and writing each $x, y>0$ in the form

$$
x=e^{s}, \quad y=e^{t},
$$

for some $s, t \in \mathbb{R}$ gives us, using properties of exponential functions,

$$
\log \circ g\left(e^{s+t}\right)=\log \circ g\left(e^{s}\right)+\log \circ g\left(e^{t}\right), \quad s, t \in \mathbb{R},
$$

which means that function $\alpha=\log \circ g \circ \exp$ is additive. This finishes the proof. 
We reformulate this result in terms of the harmonic mean and the Cauchy difference in the following.

REMARK 4. A function $\alpha: \mathbb{R} \rightarrow \mathbb{R}$ is additive if and only if

$$
C_{\alpha \circ \log }(x, y)=\alpha \circ \log \left(\frac{2}{H(x, y)}\right), \quad x, y>0 .
$$

Remark 1, Remark 2, Remark 3 and Remark 4lead to the question on the relation between different types of Cauchy differences or beta-type functions and means, which will be treated in our next paper.

\section{References}

[1] Heuvers K.J., Another logarithmic functional equation, Aeq. Math. 58 (1999), 260-264.

[2] Himmel M., Matkowski J., Homogeneous beta-type functions, J. Math. Inequal. To appear.

[3] Kannappan Pl., Functional equations and inequalities with applications, Springer Monographs in Mathematics, Springer, New York, 2009.

Faculty of Mathematics

Computer Science and Econometrics

University of Zielona Góra

SZAFRANA $4 \mathrm{~A}$

65-516 Zielona Góra

POLAND

e-mail: himmel@mathematik.uni-mainz.de 